\title{
Advanced cardiovascular physiology studied by applying the equivalent electronic circuit
}

\author{
M. Jelenc ${ }^{1}$, T. Podnar ${ }^{1}$, F. Runovc ${ }^{2}$ and M. Kordas $\breve{s}^{3}$ \\ 'University Hospital of Paediatrics, Ljubljana, Slovenia \\ ${ }^{2}$ Faculty of Natural Science and Engineering, Ljubljana, Slovenia \\ ${ }^{3}$ Institute of Pathophysiology, Ljubljana, Slovenia
}

\begin{abstract}
The equivalent electronic circuit, developed to simulate cardiovascular physiology, is upgraded to incorporate negative feed-back loops. In this way homeostasis of the arterial pressure is simulated in left ventricular failure both if the feed-back loops are disconnected from, or connected to, their target organs. The results show that in left ventricular failure homeostasis strongly supports the cardiovascular system both by modulating Starling mechanism(s) of the heart and by modulating the tone of the venous system.
\end{abstract}

\section{Introduction}

There are various approaches in modelling methodology for physiology and medicine (reviewed in [1]). However, recently it has been reported that various physiological processes can be simulated by constructing equivalent electronic circuits $[2,3]$. This approach was extended and applied to the cardiovascular system [4]. By upgrading the equivalent electronic circuit the diastolic functions of the heart muscle were investigated. By suitable adjustment of various parameters determining the function of the right and left ventricle possible mechanisms of compensation were studied [5].

The aim of the present study is to investigate whether it is possible to include, into the equivalent circuit of the cardiovascular system, a homeostatic mechanism operating by negative feed-back. To make the system not too complex only three negative feed-back loops (by sensing changes in mean arterial pressure) controlling three target organs (the systemic venous reservoir and the contractility of the right and left ventricle) are designed. 


\section{Simulations in Biomedicine $V$}

By using this upgraded circuit various normal and pathologic conditions in the cardiovascular system can be studied both under homeostatic control and if homeostasis is abolished.

In this investigation left ventricular failure (negative intrathoracic pressure not included) is simulated in two conditions: if the feed-back loops are disconnected from, or connected to, their target organs.

\section{Methods}

The simulations are performed by using Electronics Workbench, Personal version 5.12 [6]. The equivalent electronic circuit is a highly upgraded (i. e. including feed-back loops) version of that described in [4, 5]. Further minor modifications: duration of systole: $200 \mathrm{~ms}$. Time constant of ventricular contraction and relaxation: $100 \mathrm{~ms}$ and $50 \mathrm{~ms}$, respectively.

Results are expressed graphically as time course of various electrical variables. They are the so-called equivalent variables to those in the simulated physiological system (Table 1). In these simulations not the electrical terms (charge, current, voltage, etc.) but only their physiological equivalents as defined in Table 1 (blood volume, blood flow and cardiac output, pressure, etc.) are used.

Table 1: Equivalent quantities and arbitrary units in simulation of cardiovascular system

\begin{tabular}{|l|l|l|l|}
\hline electronic circuit & arbitrary unit(s) & $\begin{array}{l}\text { cardiovascular } \\
\text { system }\end{array}$ & arbitrary unit(s) \\
\hline \hline voltage & $1 \mathrm{~V}$ & pressure & $10 \mathrm{~mm} \mathrm{Hg}$ \\
\hline $\begin{array}{l}\text { ground potential } \\
\text { (reference for } \\
\text { voltage measure- } \\
\text { ment) }\end{array}$ & $0 \mathrm{~V}$ & $\begin{array}{l}\text { atmospheric pres- } \\
\text { sure (reference } \\
\text { for pressure measu- } \\
\text { rement) }\end{array}$ & $0 \mathrm{~mm} \mathrm{Hg}$ \\
\hline current & $1 \mu \mathrm{A}=60 \mu \mathrm{As} / \mathrm{min}$ & blood flow & $100 \mathrm{~mL} / \mathrm{s}=6000 \mathrm{~mL} / \mathrm{min}$ \\
\hline resistance & $10 \mathrm{~V} / 1 \mu \mathrm{A}=10 \mathrm{M \Omega}$ & resistance & $100 \mathrm{~mm} \mathrm{Hg} / 100 \mathrm{~mL} / \mathrm{s}=1 \mathrm{U}$ \\
\hline capacitance & $1 \mu \mathrm{F}=1 \mu \mathrm{As} / 1 \mathrm{~V}$ & capacitance & $100 \mathrm{~mL} / 10 \mathrm{~mm} \mathrm{Hg}$ \\
\hline charge & $1 \mu \mathrm{As}$ & volume & $100 \mathrm{~mL}$ \\
\hline
\end{tabular}

\subsection{Approach to the simulation of control mechanisms}

It should be pointed out that the operation of the cardiovascular system is defined by various parameters (e. g. blood volume, capacitance and resistance of blood vessels, contractility of the heart, etc.). If active, many variables (e. $g$. arterial pressure, cardiac output, etc.) can be recorded and studied. A disturbance (change of a parameter) results in a change of variables; these are then corrected (compensated) by homeostatic, negative feed-back mechanisms. Thus, e. g. in animal experiment exercise (decrease in peripheral resistance) 
results in an initial slight increase in cardiac output and decrease in mean arterial pressure. Consequently, by baroreflex (negative feed-back) mechanisms the parameters of target organs (the heart, arteries and veins) are reset to another value. Consequently, cardiac output is strongly increased until mean arterial pressure is almost normal $[7,8]$.

Similar principles can be applied also to the present simulations. The negative feed-back should comprise the pressure sensing mechanism and its actions on target organs.

\subsection{Principles of simulation}

The analog equivalent circuit (Fig. 1, cf. $[4,5]$ ) simulates the cardiovascular system and its controlling feed-back loops. For the sake of clear description and easy comparison with previous circuits [3 - 5] the same or similar acronyms are used as listed below:

$\begin{array}{ll}\text { AoP } & \text { aortic pressure } \\ \text { CO } & \text { cardiac output } \\ \text { CVV } & \text { "contractible" volume of veins } \\ \text { LAtP } & \text { left atrial pressure } \\ \text { LV } & \text { left ventricle } \\ \text { MAoP } & \text { mean arterial pressure (time integral of AoP) } \\ \text { RAtP } & \text { right atrial pressure } \\ \text { RV } & \text { right ventricle }\end{array}$

Essentially, the feed-back loop starts as a mechanism recording the arterial pressure. Its changes are then, as a negative feed-back message, transferred to two target organs: heart muscle and systemic veins. Thus not only the venous tone, but also the LV and RV contractility is modulated.

In simulating LV failure, only RV contractility is modulated. The procedure is as follows: AoP is integrated against time to obtain its mean pressure (MAoP). If the latter is at resting level (about $98 \mathrm{~mm} \mathrm{Hg}$ ) both the venous tone and contractility of RV and LV are at their resting level. If, e. g. LV contractility is decreased (LV failure, parameter change), AoP and consequently MAoP decrease. Through negative feed-back the function of target organs is modulated: venous tone is increased (decreasing the "contractible" volume of veins, CVV) and contractility of RV is increased. Cardiac output is increased until MAoP is slightly below $98 \mathrm{~mm} \mathrm{Hg}$. The extent of MAoP change thus corresponds to the "error voltage" in voltage-clamping the membrane potential during neuromuscular transmission $[9,10]$. This means that MAoP change is the smaller the larger is the gain in the feed-back circuit. Effectively, the present circuit operates as a "MAoP-clamp".

Data on operational amplifiers: A2: gain 1. It is used as an isolation unit between various parts of the circuitry. A8: gain 2. A1: gain 10. AA2: gain 1 (output voltage range $25 \mathrm{~V} /-25 \mathrm{~V}$ ). 


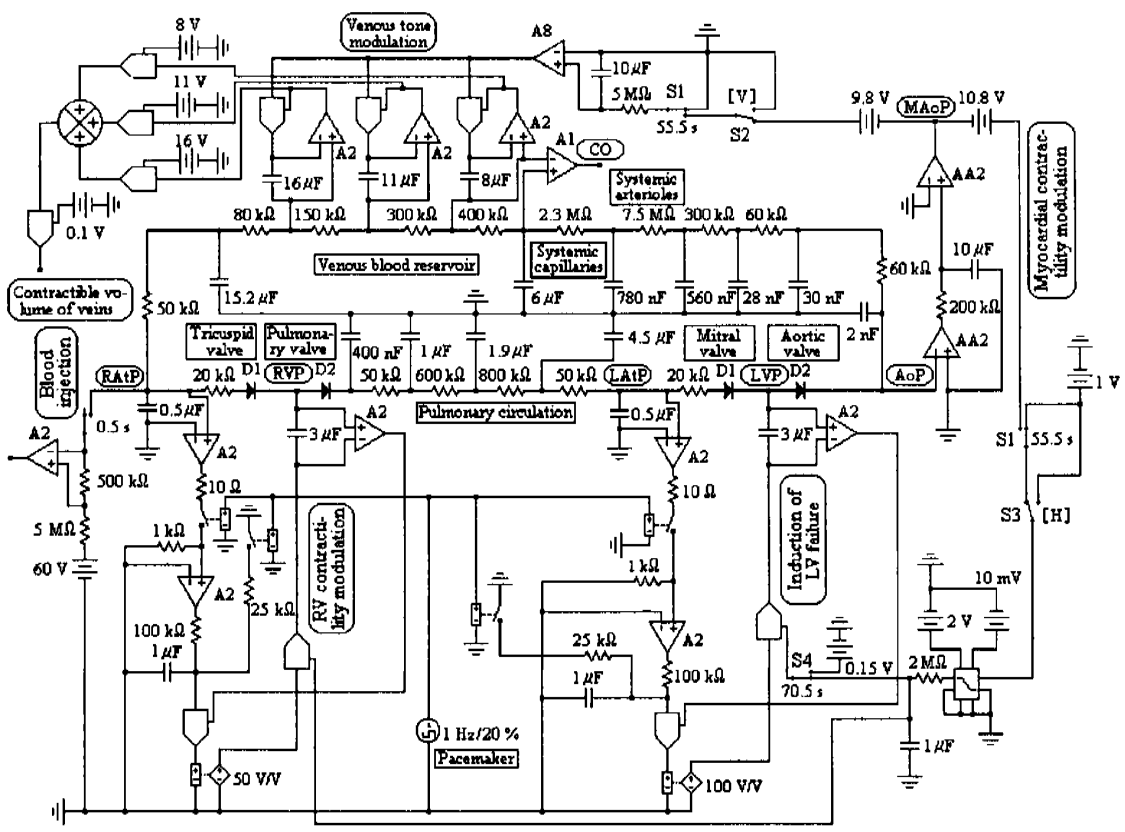

Fig. 1. Electronic circuit of the upgraded cardiovascular system. Equivalents to anatomic structures indicated by anatomic terms. The points to measure variables of the cardiovascular system indicated by their acronyms. Voltage multipliers in RV and LV amplifying circuit allow gain to be modulated according to the voltage generated in the feed-back loops. The negative feedback: AoP is time-integrated $\left(\tau_{1}=2 \mathrm{~s}\right)$ to obtain MAoP. This voltage is fed into the venous tone modulation subcircuit and (in this simulation only) into the RV modulation subcircuit. The venous tone modulation subcircuit: Offset battery $(9.8 \mathrm{~V})$, switches $\mathrm{S} 1$ and $\mathrm{S} 2$ and a time-delay circuit $\left(\tau_{2}=50 \mathrm{~s}\right)$. The resulting voltage is amplified (open-loop gain: 2 ) and fed into the capacitors simulating the venous blood reservoir. The included voltage multipliers assure that volume of veins (i. e. CVV) is always larger from 0 . The myocardial modulation subcircuit: Offset battery $(10.8 \mathrm{~V})$, switches $\mathrm{S} 1$ and $\mathrm{S} 3$, a voltage clipper and a time-delay circuit $\left(\tau_{3}=2 \mathrm{~s}\right)$. Its voltage is fed into the voltage multiplier modulating the gain in RV (open-loop gain: 1). In LV circuit modulation is replaced by a "failure" circuit. Feed-back loops closed: The steady state of variables maintaining venous tone and contractility of ventricles (time constants $\tau_{1}, \tau_{2}$ and $\tau_{3}$ ) achieved in about $25 \mathrm{~s}$ and $10 \mathrm{~s}$, respectively, after an instantaneous parameter change. Switches S1 and S4: Time-delay switches. The former connects the feed-back loops to its targets at $55.5 \mathrm{~s}$. The latter induces LV failure at $70.5 \mathrm{~s}$. Switches S2 and S3: Key operated ("V": veins; "H": heart); feed-loops can be connected to, or disconnected from, its targets. 


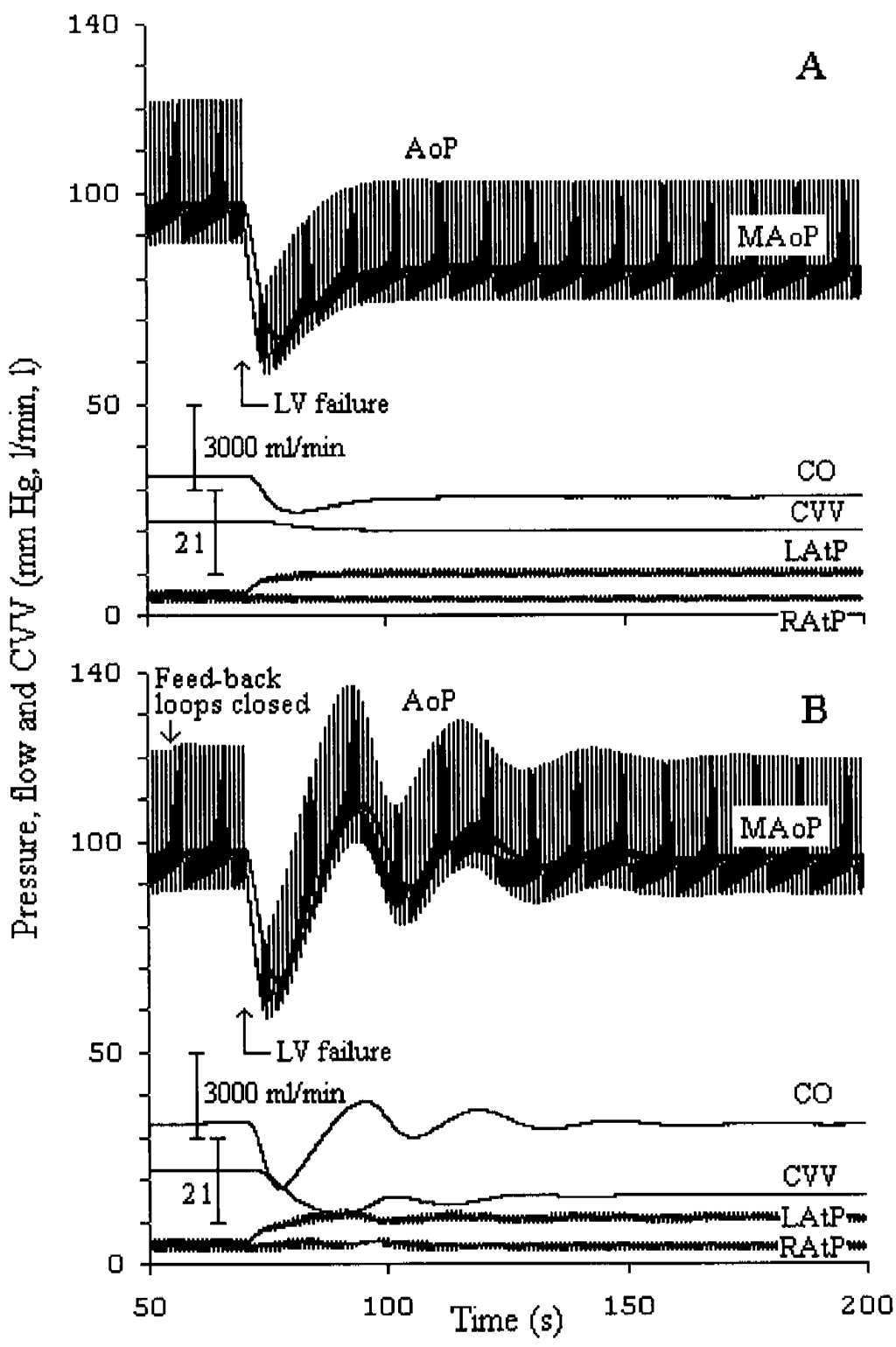

Fig. 2. The time course of various variables after LV failure. A: Feed-back loops open. AoP, MAoP and CO initially decreased, followed by a rapid, but not complete recovery at about $100 \mathrm{~s}$. CVV slightly decreased. LAtP increased, RAtP not changed. B: Feed-back loops closed. AoP, MAoP and CO initially decreased followed by a rapid, oscillatory response with slight overshoots. Almost complete recovery at about $150 \mathrm{~s}$. CVV decreased almost by $50 \%$. LAtP increased slightly above the level shown in A. 


\subsection{Simulation procedure}

After analysis is started about $45 \mathrm{~s}$ is allowed to establish the steady state of all variables. Then they are recorded for the time interval from $50 \mathrm{~s}$ to $200 \mathrm{~s}$. At $55.5 \mathrm{~s}$ the feed-back loops are closed, i. e. connected to their targets, the venous tone and myocardial contractility modulation points. At this instant a minute change in AoP can be observed (cf. Fig. 2, arrow) due to the fact that MAoP (time integral of AoP) is not absolutely constant. The parameter change (disturbance) is introduced at $70.5 \mathrm{~s}$ and is maintained until end of the simulation $(200 \mathrm{~s})$.

If required, the feed-back loop can be interrupted. If so, the venous tone and myocardial contractility are maintained at their resting level.

Measurements are performed as described [3-5].

\section{Results}

Parameter change: decrease of LV contractility by $85 \%$.

The effects of LV failure are shown in Fig. 2A and 2B as time course of AoP, MAoP, CO, CVV, LAtP and RAtP.

\subsection{A: Feed-back loops open}

AoP, MAoP and CO initially decreased, followed by a rapid, but not complete recovery. CVV slightly decreased. LAtP increased, RAtP not changed. Steady state established at about $100 \mathrm{~s}$ of simulation time.

\subsection{B: Feed-back loops closed}

AoP, MAoP and $\mathrm{CO}$ initially decreased as in A, followed by a rapid, oscillatory response with slight overshoots. Almost complete recovery. CVV decreased almost by $50 \%$. LAtP increased slightly above the level shown in A, RAtP not changed. Steady state established at about $150 \mathrm{~s}$ of simulation time.

\section{Discussion}

Apart from LV failure described above also many other disturbances have so far been successfully simulated (exercise, haemorrhage, blood volume expansion, aortic and mitral insufficiency, patent ductus arteriosus, ventricular septal defect) both under homeostatic control and if homeostasis is abolished. In some of these experiments also negative intrathoracic pressure and its respiratory variations were successfully simulated. 


\section{1. General observations}

If feed-back loops are closed it is MAoP which defines the volume of CVV. If the former is decreased, the latter is also decreased.

If feed-back loops are open, MAoP does not define the volume of CVV. In this condition CVV is defined by total blood volume and blood volume distribution within the vascular circuit.

\section{2. Specific observations}

If feed-back loops are open, LV failure results in a primary decrease in CO and blood volume redistribution. The volume of the systemic circuit (predominantly systemic veins) is decreased and the volume of the pulmonary circuit is increased. LAtP is increased and via Starling mechanisms the force of systolic contraction of $\mathrm{LV}$ is improved, leading to a partial recovery of $\mathrm{CO}, \mathrm{AoP}$ and MAoP.

If feed-back loops are closed, the initial MAoP decrease results in an increased tone of systemic veins and increased contractility of RV. These two mechanisms strongly support the diastolic filling of the failing LV, resulting in an almost normal $\mathrm{CO}, \mathrm{AoP}$ and MAoP. However, this compensation is achieved at the cost of severe pulmonary congestion. These observations are almost identical to those achieved by manual compensation of LV failure, described in [5].

It seems that LV simulations described above (compensation by feed-back mechanisms) and by manual adjustments of cardiovascular parameters [5] are very similar to changes observed in man in cardiac infarction.

\section{References}

[1] Carson, E. \& Cobelli, C., (eds.), Modelling methodology for physiology and medicine, Academic Press, San Diego, 2001.

[2] Rupnik, M., Runovc, F. \& Kordaš, M., The use of equivalent electronic circuits in simulating physiological processes, IEEE Transactions Educ 44, pp. 384-389, 2001.

[3] Bošnjak, R. \& Kordaš, M., Circulatory effects of internal jugular vein compression: a computer simulation study, Med. Biol. Eng. Comput. 40, 423-431,2002.

[4] Rupnik, M., Runovc, F., Sket, D. \& Kordaš, M., Cardiovascular physiology: Simulation of steady state and transient phenomena by using the equivalent electronic circuit, Comput. Methods Programs Biomed. 67, pp. 1-12, 2002.

[5] Podnar, T., Runovc, F. \& Kordaš, M., Simulation of cardiovascular physiology: the diastolic function(s) of the heart. Computers in Biology and Medicine, 32, pp. 363 - 377, 2002.

[6] Interactive Images Technologies Ltd.; http://www.interactiv.com

[7] Topham, S. \& Warner, H. R., The control of cardiac output during exercise, 


\section{Simulations in Biomedicine $V$}

Physical bases of circulatory transport. Regulation and exchange, ed. E. B. Reeve \& A. C. Guyton, W. B. Saunders, Philadelphia, 1967, 77 - 90.

[8] Berne, R. M. \& Levy, M. N., Cardiovascular physiology $7^{\text {th }}$ edn., Mosby, St. Louis, 1997, pp. 55-81.

[9] Kordaš, M., Melik Živa, Peterec, D. \& Zorec, R., The voltage-clamp apparatus assisted by a 'current pump'. $J$ Neurosci Methods, 26, pp. 229232, 1989.

[10] Kordaš, M., Melik Živa, Peterec, D. \& Zorec, R., Current pump-assisted voltege-clamp apparatus', Electrophysiology and microinjection. Methods in Neurosciences, ed. P. M. Conn, Academic Press, New York, 1991, 94 102.

[11] Kusumoto, F., Cardiovascular Disorders: Heart Disease, Pathophysiology of disease. An introduction to clinical medicine, ed. S. J. McPhee, V. R. Lingappa, W. Ganong \& J. D. Lange, Lange Medical Books/McGraw-Hill, New York, 2000, $222-257$. 